\title{
Correlated many-electron states in a quantum dot containing a single magnetic impurity
}

\author{
Nga T. T. Nguyen* and F. M. Peeters ${ }^{\dagger}$ \\ Departement Fysica, Universiteit Antwerpen, Groenenborgerlaan 171, B-2020 Antwerpen, Belgium
}

(Received 14 March 2007; revised manuscript received 6 June 2007; published 16 July 2007)

\begin{abstract}
The few-electron states of a parabolic confined quantum dot is studied in the presence of a magnetic ion. The electrons interact with a single magnetic ion $\left(\mathrm{Mn}^{2+}\right)$ which is located in the quantum dot at arbitrary position. The local Zeeman spin splitting is calculated for different numbers of electrons $\left(N_{e}=1,2,3, \ldots, 10\right)$. The effect of the position of the magnetic ion on the energy levels (both ground state and excited states) is investigated. We calculate the heat capacity as a function of temperature, the position of the impurity, and the Coulomb strength.
\end{abstract}

DOI: 10.1103/PhysRevB.76.045315

PACS number(s): 73.21.La, 75.30.Hx, 75.50.-y

\section{INTRODUCTION}

Quantum dots ${ }^{1}$ have attracted a lot of attention due to their interesting physics, such as transformations of the multielectron ground state as a function of magnetic field resulting in, for instance, switching of total spin and total angular momentum of the system. ${ }^{2}$ Electrons in a quantum dot interact with each other via the Coulomb potential and with the lateral harmonic confinement potential. The relative interaction strength, $\lambda$, that can be varied in a wide range ${ }^{3}$ is given by the ratio of the effective width of the confinement, $l_{0}$, and the Bohr radius of the host semiconductor, $a_{B}$ $=4 \pi \epsilon_{0} \epsilon \hbar^{2} / m^{*} e^{2}$ as follows:

$$
\lambda=\frac{e^{2} / 4 \pi \epsilon_{0} \epsilon l_{0}}{\hbar \omega_{0}}=\frac{l_{0}}{a_{B}} .
$$

Large $\lambda$ implies strong Coulomb interaction, and vice versa. In this paper, we focus on a few values of $\lambda$ as long as the gap between the single-particle energy spacings $\hbar \omega_{0}$ is large enough as compared to the Coulomb interaction energy.

Magnetic doping of quantum dots based on II-VI semiconductors has been demonstrated. ${ }^{4}$ More recently, a quantum dot doped with a single magnetic ion was realized. ${ }^{5,6}$ Such a system allows the control of the interaction between individual charge carriers and a single magnetic atom. This is expected to be important for future spintronic and quantum computing devices.

Theoretical studies on the interaction of electrons in diluted magnetic semiconductor (DMS) quantum dots were presented by several groups. ${ }^{7-9}$ Those publications addressed the case of a large number of magnetic impurities allowing for a mean-field representation of the local magnetization of the magnetic ions. Here we consider the opposite case in which only a single magnetic ion is present in the quantum dot.

This paper explores the electronic properties of the ground state of $N_{e}$ interacting electrons confined by a parabolic potential containing a single magnetic impurity $\mathrm{Mn}$ at zero magnetic field. This is the situation recently studied in Ref. 10, where the authors found a local Zeeman spin splitting independent of the position of the impurity for $N_{e}$ $=1,2, \ldots, 6$ including only $s, p$, and $d$ shells. In the preceding calculations, the magnetic impurity was fixed at the position $\left(l_{0}, 0\right)$ and the Coulomb strength was kept at a specific value $\lambda=0.5$. In this paper, we extend those calculations to a quantum dot containing up to ten electrons where the Coulomb interaction strength will be varied and the single magnetic impurity may be located at an arbitrary position inside the dot. We enlarge the size of the Hilbert space, as compared to Ref. 10, by including higher levels, beyond the $s, p$, and $d$ shells. Besides the ground-state energy, we will also investigate thermodynamic properties as the specific heat.

The paper is organized as follows. In Sec. II we present our model and the approach to solve it numerically. In Secs. III and IV we present the ground-state energy and the excited states for up to ten electrons. Section $\mathrm{V}$ is reserved for the heat capacity calculations and our conclusions are presented in Sec. VI.

\section{MODEL AND METHOD}

We refer to the review paper of Ref. 11 for general information on mostly bulk DMS and to Ref. 12 for recent papers on many-particle states in Mn-doped quantum dots.

We study the magnetic exchange interaction behavior in quantum dots containing $N_{e}$ electrons with spin $1 / 2$ and effective mass $m^{*}$ at positions $\overrightarrow{r_{i}}=\left(x_{i}, y_{i}\right)$ and momenta $\overrightarrow{p_{i}}$ $=-i \hbar \vec{\nabla}_{i}$ with a single magnetic ion (e.g., $\mathrm{Mn}^{2+}$ ) with spin $5 / 2$ located at position $\vec{R}=(X, Y)$. Those electrons move in a twodimensional (2D) plane and are confined by a parabolic confinement potential. The Hamiltonian describing this problem is

$$
\begin{aligned}
\hat{H}= & \sum_{i=1}^{N_{e}}\left[-\frac{\hbar^{2}}{2 m^{*}} \vec{\Delta}_{i}+V_{C}\left(\overrightarrow{r_{i}}\right)\right]+\frac{1}{2} \sum_{i \neq j}^{N_{e}} V\left(\vec{r}_{i}-\vec{r}_{j}\right) \\
& -J_{c}^{2 \mathrm{D}} \sum_{i=1}^{N_{e}} \vec{M} \cdot \overrightarrow{S_{i}} \delta\left(\overrightarrow{r_{i}}-\vec{R}\right)
\end{aligned}
$$

with

$$
V_{C}\left(\overrightarrow{r_{i}}\right)=\frac{1}{2} K \vec{r}_{i}^{2},
$$

the confinement potential with $K=m^{*} \omega_{0}^{2}$ the confinement strength, and $\omega_{0}$ is the confinement frequency that introduces the confinement length $l_{0}=\sqrt{\hbar / m^{*} \omega_{0}}$. A parabolic confine- 
ment potential is the standard approximation for lithographically defined quantum dots embedded in a two-dimensional electron gas. The Mn-doped CdTe dots of Refs. 4 and 5 are self-assembled dots which are three-dimensional structures with a more hard-wall-like confinement potential. Often, the height of those dots is much smaller than their diameter and an effective 2D approach is still reasonable. A hard-wall confinement potential leads to qualitative differences ${ }^{13}$ but the important physics we are interested in is not influenced by the precise shape of the confinement potential.

The first two terms in Hamiltonian (2) describe the oneelectron Hamiltonian problem (the kinetic energy and the harmonic terms) and the third term is the Coulomb interaction between the electrons $V\left(\overrightarrow{r_{i}}-\overrightarrow{r_{j}}\right)=e^{2} / 4 \pi \epsilon_{0} \epsilon\left|\overrightarrow{r_{i}}-\overrightarrow{r_{j}}\right|$ with $e$ and $\epsilon$ being the electron charge and dielectric constant, respectively. The last term gives the electron-magnetic impurity interaction and is responsible for the flipping of the spins $\vec{S}_{i}$ of the electrons and the spin $\vec{M}$ of the single Mn ion (spin size $\mathbf{M}=\frac{5}{2}$ ) and the scattering of electrons to different orbitals. The exchange interaction strength is given by the prefactor $J_{c}^{2 \mathrm{D}}$ which, as in Ref. 8, is taken equal to $1.5 \mathrm{eV} \AA^{2}$, which is independent of the lateral dimension of the quantum dot. This scattering term is similar to the term in Kondo and Anderson models, see, e.g., Ref. 14.

In the previous calculations ${ }^{10}$ the magnetic impurity was fixed at the location $\left(l_{0}, 0\right)$ inside the quantum dot. Here we will release this restriction and investigate the dependence of the quantum properties on the position of the Mn ion.

The single-particle problem in a parabolic potential defines a complete basis set of Fock-Darwin orbitals $\phi_{n l}(\vec{r})$ and spin functions $\chi_{\sigma}(\vec{S})$ as follows:

$$
\phi_{n l s}(\vec{r}, \vec{S})=\varphi_{n l}(\vec{r}) \chi_{\sigma}(\vec{S})
$$

where the Fock-Darwin orbitals are

$$
\varphi_{n l}(\vec{r})=\frac{1}{l_{0}} \sqrt{\frac{n !}{\pi(n+|l|) !}}\left(\frac{r}{l_{0}}\right)^{|l|} e^{i l \theta-r^{2} / 2 l_{0}^{2}} L_{n}^{|l|}\left(r^{2} / l_{0}^{2}\right),
$$

$(n, l, \sigma)$ are radial, azimuthal (angular momentum), and spin quantum numbers $(+1$ for the spin-up projection and -1 for the spin-down projection), respectively. $L_{n}^{|l|}(r)$ is the Laguerre polynomial. The energy levels of the single-particle states are

$$
E_{n l s}=\hbar \omega_{0}(2 n+|l|+1) \text {. }
$$

We denote each single Fock-Darwin state by an index $i$ $=\{n, l\}$, which includes two quantum numbers $n=0,1,2, \ldots$. and $l=0, \pm 1, \pm 2, \ldots$.

Following Ref. 10 the second-quantized form of the above Hamiltonian is obtained in the following basis functions (4):

$$
\begin{aligned}
\hat{H}= & \sum_{i, \sigma} E_{i, \sigma} c_{i, \sigma}^{+} c_{i, \sigma}+\frac{1}{2} \sum_{i j k l}^{\sigma \sigma^{\prime}}\left\langle i, j\left|V_{0}\right| k, l\right\rangle c_{i, \sigma}^{+} c_{j, \sigma^{\prime}}^{+} c_{k, \sigma^{\prime}} c_{l, \sigma} \\
& -\sum_{i j} \frac{J_{i j}(R)}{2}\left[\left(c_{i, \uparrow}^{+} c_{j, \uparrow}-c_{i, \downarrow}^{+} c_{j, \downarrow}\right) M_{z}+c_{i, \uparrow}^{+} c_{j, \downarrow} M^{-}\right. \\
& \left.+c_{i, \downarrow}^{+} c_{j, \uparrow} M^{+}\right] .
\end{aligned}
$$

Ket vector $|i, \sigma\rangle$ indicates a quantum state with a set $(n, l, \sigma)$ being radial, azimuthal, and $z$-component spin quantum numbers, respectively. Creation (annihilation) operator $c_{i \sigma}^{+}\left(c_{i \sigma}\right)$ acts on a ket vector $|i, \sigma\rangle$ and creates (annihilates) a particle with the quantum set $(n, l, \sigma) . V_{0}=e^{2} / 4 \pi \epsilon_{0} \epsilon l_{0}$ is the prefactor in the Coulomb matrix elements and was previously calculated by Tsiper. ${ }^{15} M_{z}, M^{+}$, and $M^{-}$are the $z^{-}$and raisingand lowering-spin operators of the magnetic moment of the ion.

We consider quantum states in the language of nuclei shells but defined for a 2D quantum system. Those orbitals are in the same shell provided that (a) electrons occupying those orbitals have the same energy level, and (b) their respective azimuthal quantum numbers have the same parity.

Electrons in different orbitals can interact with the magnetic ion through the contact exchange interaction. The last term in Hamiltonian (2) that describes the electron-ion-Mn exchange interaction can be rewritten as follows:

$$
H_{e-\mathrm{Mn}}=-J_{c}^{2 \mathrm{D}} \sum_{i j \sigma \sigma^{\prime}} J_{i j}(R) \vec{S}_{\sigma, \sigma^{\prime}} \cdot \vec{M} c_{i, \sigma}^{+} c_{j, \sigma^{\prime}},
$$

with

$$
J_{i j}(R)=J_{c}^{2 \mathrm{D}} \varphi_{i}^{*}(R) \varphi_{j}(R)
$$

the exchange matrix elements. Each of those elements is a product of two single-particle wave functions of those two exchanged quantum states calculated at position $\vec{R}$ of the impurity. They build up a submatrix called the exchange interaction matrix. The size of this matrix is determined by the number of orbitals that are taken into account in our calculations. For instance, if we include all six orbitals of $s, p$, and $d$ shells (as in Ref. 10) the size of this matrix would be $6 \times 6$.

Those two quantum numbers: total angular momentum $L$ and $z$ component of the total spin $S_{z}$ of the electrons are not conserved due to the presence of the magnetic ion with specific spin $\vec{M}$. Recall that without the magnetic impurity those numbers would be conserved and they are good quantum numbers reducing considerably the Hamiltonian matrix. Ground-state properties of that problem can be described in the subspace $\left(L, S_{z}\right)$.

Because those quantities are not conserved due to the coupling of different single orbitals we need the entire Hilbert space to build up the Hamiltonian matrix. After diagonalization of this Hamiltonian we obtain the energy spectrum. To construct those elements of the Hamiltonian we use the configuration interaction method to expand the wave function in the single-electron basis as follows: 


$$
\Psi\left(\overrightarrow{x_{1}}, \overrightarrow{x_{2}}, \ldots, \overrightarrow{x_{N_{e}}^{*}}, \vec{M}\right)=\sum_{k=1}^{N_{C}} C_{k} \Psi_{k},
$$

where $\Psi_{k}$ is the $k$ th state of the wave function determined by $N_{e}$ electrons with $N_{e}$ different sets of quantum numbers $(n, l, \sigma)$ and the single scatterer with one of the six states of the projection of the spin. $\vec{x}_{i}^{*}=\left(\vec{r}_{i}, \vec{S}_{i}\right)$ stands for the coordinates and spin of a single electron. In the second quantization representation, the state $\Psi_{k}$ can be translated into a ket vector $|k\rangle$ grouping total $N_{e}$ electrons into $N \uparrow$ electrons with $z$ component of spin up and $N \downarrow=N_{e}-N \uparrow$ electrons with $z$ component of spin down as follows:

$$
\Psi_{k} \Rightarrow|k\rangle=\left|c_{i_{1}}^{+}, c_{i_{2}}^{+}, \ldots, c_{i_{N \uparrow}}^{+}\right\rangle\left|c_{j_{1}}^{+}, c_{j_{2}}^{+}, \ldots, c_{j_{N \downarrow}}^{+}\right\rangle\left|M_{z}\right\rangle .
$$

Here $i_{1} / i_{N \uparrow}$ and $j_{1} / j_{N \downarrow}$ are the indices of single states for which each index is set by two quantum numbers (radial and azimuthal quantum numbers), as mentioned above.

The number of possible configurations $N_{C}$ depends on the number of electrons, $N_{e}$, the number of single orbitals, $N_{S}$, and the size of the spin, $\mathbf{M}$, of the magnetic ion $\mathrm{Mn}$. That $N_{e}$ electrons interact with the single magnetic impurity ion via the contact interaction implies that those different single orbitals couple with one another. As a consequence states with different total angular momenta and/or different total spin of electrons can scatter to other states. As a result, one needs to take into account all states generated from the three specific quantum numbers: $N_{e}, N_{S}$, and $M$ (here we take $M=5 / 2$ ).

Consequently, the $N_{e}$-electron quantum dot in the presence of the magnetic impurity has a Hamiltonian matrix that is six times larger than that in the case without the magnetic ion when including the same number of single-particle orbitals. This limits the number of single orbitals, $N_{S}$, that can be included to obtain results as accurate as possible.

As an example, let us consider the three-electron quantum dot. If one includes only $s$ and $p$ shells, it means that only three orbitals along with two possibilities of the electron spin $1 / 2$ are included and one would have $N_{C}=120\left(6 \times C_{6}^{3}\right)$ configurations. If one includes $s, p$, and $d$ shells one would have $N_{C}=1320\left(6 \times C_{12}^{3}\right)$ configurations. The number of configurations would be $N_{C}=166320$ if one includes 28 orbitals.

We may recall that in the $s$ shell one has two sets of quantum numbers being $(0,0,1)$ and $(0,0,-1)$ with 1 and -1 the up- and down-z-component quantum numbers of electron spin $(1 / 2)$. In $p$ shell one has four respective sets being $(0,-1,1),(0,-1,-1),(0,1,1),(0,1,-1)$, and so on.

Here, we consider a magnetic impurity with spin size 5/2 that is typical for a Mn ion in, e.g., a II-VI (Cd,Mn)Te quantum dot. We can use the following typical parameters: effective width $l_{0}=26.45 \AA$, effective Bohr radius $a_{B}=2 \times l_{0}$ $=52.9 \AA$, effective mass $m^{*}=0.106 m_{e}, \epsilon=10.6$, quantum dot thickness $W=2 \mathrm{~nm}$, and confinement frequency (energy spacing) $\omega_{0}=51.32 \mathrm{meV}$.

\section{GROUND-STATE ENERGY}

\section{A. Convergency}

As compared to the earlier study, ${ }^{10}$ we included higher levels (more electronic shells). Increasing the number of

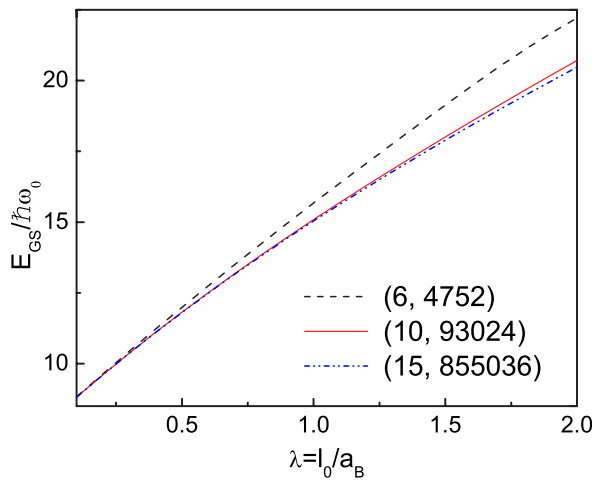

FIG. 1. (Color online) Convergency of the ground-state energy of a five-electron quantum dot. The magnetic impurity is located at $(0,0)$.

single-energy levels will tend the problem to asymptotically approach the exact one, but in a different way from the problem when no impurity is present. The latter problem can be treated by the same exact diagonalization method (see, for instance, Ref. 16 and references therein) using the quantum number $\left(L, S_{z}\right)$ subspace technique.

Our problem concerned with a more generic picture since we consider many more possibilities of configurations due to the presence of the magnetic ion with spin 5/2 that induces coupling between states with different quantum numbers $\left(L, S_{z}\right)$. This results in a rapid increase of the number of configurations when involving more shells.

First, we investigate the convergency of our results as a function of the number of shells that are included in the numerical calculation. As an example, we show in Fig. 1 the case of $N_{e}=5$ electrons for the case that the interaction with the magnetic ion is included. For $\lambda=0.5$ the energy is 11.81758 for $N_{C}=93024$ and 11.80572 for $N_{C}=855036$, respectively. The relative difference comprises $0.1 \%$.

In Fig. 1, the first couple $(6,4752)$ means that we included six orbitals (i.e., $s, p$, and $d$ shells) and the last couple $(15,855036)$ means that the $s, p, d, f$, and $g$ shells were retained. Notice that with increasing Coulomb coupling more single-particle shells have to be included in order to obtain accurate results for the energy.

\section{B. Local Zeeman energy}

The Zeeman term contribution is calculated as being the energy difference between the quantum dot with and without the magnetic impurity as follows:

$$
\Delta_{Z}=E_{U C}-E_{C}
$$

We reproduced the results for the local Zeeman splitting as obtained recently by $\mathrm{Qu}$ and Hawrylak ${ }^{10}$ for partly filled shells for $N_{e}=1,2, \ldots, 6$ of the $s, p$, and $d$ shells in Fig. 2 by the red squares. Besides, we also show the results for some typical positions of the magnetic ion. The black circles are results for the case the magnetic ion located at the center of the dot, and the green lozenges for the magnetic ion located at $\left(\sqrt{2} l_{0}, 0\right)$. The blue triangular is representative for the case that all possible configurations of the $s, p$, and $d$ shells are 


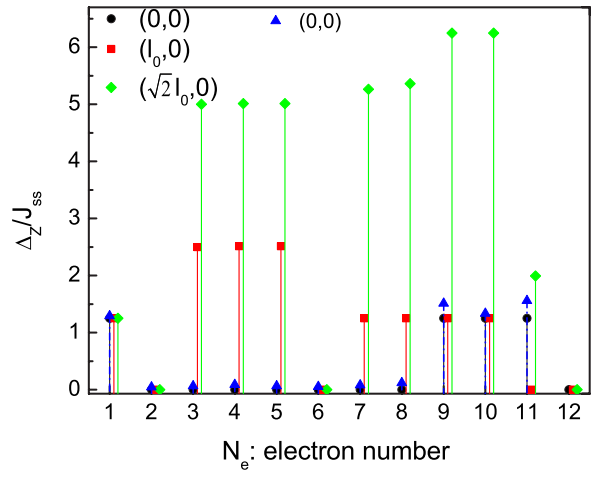

FIG. 2. (Color online) Zeeman spin splitting vs number of electrons calculated for the partially filled and for the case that all possible configurations of the $s, p$, and $d$ shells are included (blue triangles). The Coulomb strength was 0.2.

included and the magnetic ion is located at the center of the quantum dot. These results were not presented in Ref. 10. Furthermore, we found clearly that the quantitative results depend on the exact location of the magnetic impurity. The Coulomb interaction strength is chosen to be 0.2 to ensure the accuracy of the result when $N_{e}$ is increased above six electrons.

The partly filled shell calculations are done for those configurations of electrons at which there is no excitation from lower to higher electronic single-particle levels. For instance, three electrons in partly filled shells first fill up the $s$ shell by two electrons then fill up one of the four possibilities of the $p$ shell by the remaining electron. To follow this definition, we realize that 2-, 6-, and 12-electron quantum dots are special in a sense that 2 electrons fully fill the $s$ shell, 6 electrons fully fill the three orbitals (six possibilities) of the $s$ and $p$ shells, and 12 electrons fully fill the $s, p$, and $d$ shells resulting in zero Zeeman spin splitting.

\section{Dependence on the position of the magnetic ion}

The authors of Ref. 10 found the unexpected result that the exchange term $\Delta_{Z}$ was independent of the filling of the shell in a limited Hilbert space (involving only the partially filled $s, p$, and $d$ shells). If all configurations are included the $\Delta_{Z}$ slightly changed due to the presence of excitations. Additionally, they claimed that the result was independent on the position of the magnetic ion.

Our numerical calculations on the other hand show that, in general, the local Zeeman term itself depends on the position of the impurity. More specific, when we consider the partially filled $s$ and $p$ shells, the $\Delta_{Z}$ of $N_{e}=1,2, \ldots, 6$ changes consistently with changes in the $J_{s s}$ and $J_{p p}$. When all possible configurations of those shells are included, the situation changes due to the mutual interactions of the overall orbitals.

We plot in Fig. 3 the dependence of the local Zeeman energy on the position of the impurity for $1,2, \ldots$, and 6 electrons as obtained by including only the partly filled $s, p$, and $d$ shells when no excitation is taken into account. We found $\Delta_{Z}=0$ and no dependence of the local Zeeman energy of the two- and six-electron quantum dot on the position of

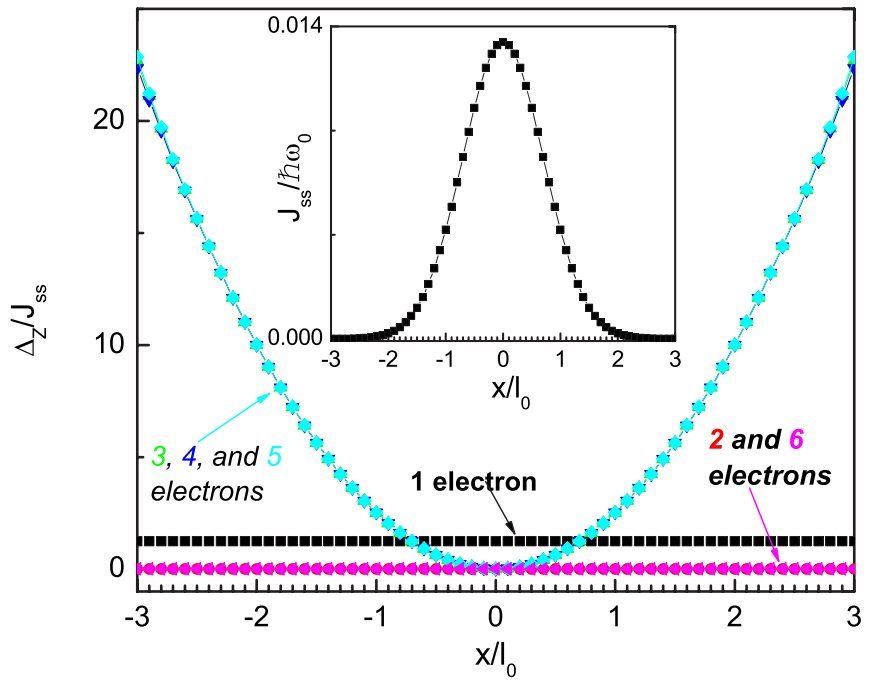

FIG. 3. (Color online) Magnetic ion position dependence of the Zeeman spin splitting for the cases of one to six electrons. $J_{s s}$ is a function of the position of the impurity as shown in the inset. The Coulomb strength is 0.2 .

the impurity. The one-electron quantum dot $\Delta_{Z}$ has only a very weak dependence. This can be explained in the following way. In the case of two- and six-electron quantum dots the $s$ and then $p$ shells were fully filled (closed shells). Therefore, there is zero contribution from the local Zeeman part to the energies of those quantum dots. For the oneelectron problem, $\Delta_{Z}$ changes consistently with the change in $J_{s s}$. This results in the ratio of the local Zeeman term to the $J_{s S}$ that is almost independent on the position of the magnetic ion.

For the three-, four-, and five-electron quantum dots we obtained the same dependence for the local Zeeman energy on the position of the impurity in case of partially filled $s$ and $p$ shells. We found zero contribution of the local Zeeman energy at position $(0,0)$. The reason is when we take into account the partially filled $s$ and $p$ shells, the exchange matrix elements describing the mutual couplings of orbitals from $s$ and $p$ shells and the exchange matrix elements between the $p$ shell themselves are zero when the Mn is at the center of the dot. We also found that the curve has a parabolic form that is also the form of the ratio of $J_{p p} / J_{s s}$ vs the position of the magnetic impurity different by a constant factor. The form of the curve changes when we include the $d$ shell orbitals.

Figure 4 shows the impurity-position dependence of the local Zeeman energy but now scaled to the confinement energy. All possible configurations were included. For $N_{e}=1$ electron, we see a peak of $\Delta_{Z}$ at $x=0$. This is because $J_{s s}$ contributes mainly to $\Delta_{Z}$ which has also a peak at $x=0$. When the number of electrons in the quantum dot increases the contributions of the exchange matrix elements from higher levels, e.g., the $p, d, f, \ldots$ shells, increase. It leads to a displacement of the peak to $x=l_{0}$, as we see for $N_{e}=3,4$, and 5 electrons.

When we move the magnetic ion far away from the center, the exchange matrix elements tend to zero. The problem is converted to an uncoupled problem because the overlap 


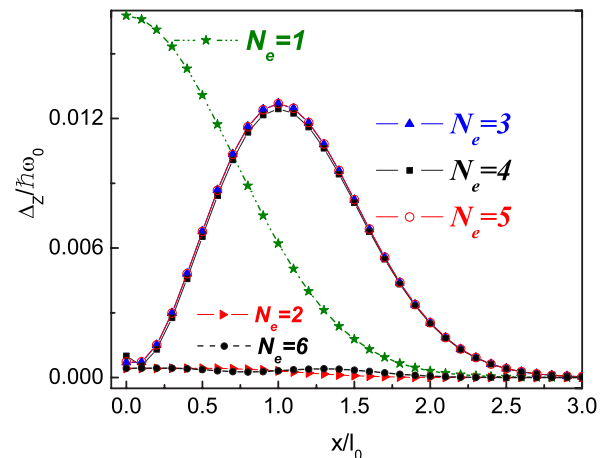

FIG. 4. (Color online) Ion-position dependence of the Zeeman spin splitting when all the possible configurations of $s, p$, and $d$ shells are included. The Coulomb strength is 0.2.

with the electron wave function decreases exponentially to zero.

When the Coulomb interaction strength, $\lambda$, approaches zero it means that the ratio $l_{0} / a_{B}$ also approaches zero. Consequently, the exchange energy, $\Delta_{Z}$, is now zero at any position of the magnetic ion, except for the center of the dot. The effect of the Coulomb interaction is to extend the region where the magnetic ion can be located where it gives a nonzero contribution of $\Delta_{Z}$ to the total energy of the system. Increasing the Coulomb interaction strength moves the maximum contribution of the local Zeeman splitting term to a position further from the origin of the dot.

\section{EXCITED STATES}

In the previous sections, we mentioned the "partially filled" concept where excitation was not taken into account in order to see the contribution of the local Zeeman term to the energy spectrum. In this section, we consider excited states where we will include higher single-particle levels. We present in Fig. 5 the energy of the first 24 energy levels for the case of $N_{e}=3$ electrons where we include 21 singleparticle orbitals. The inset is the spectrum when only the

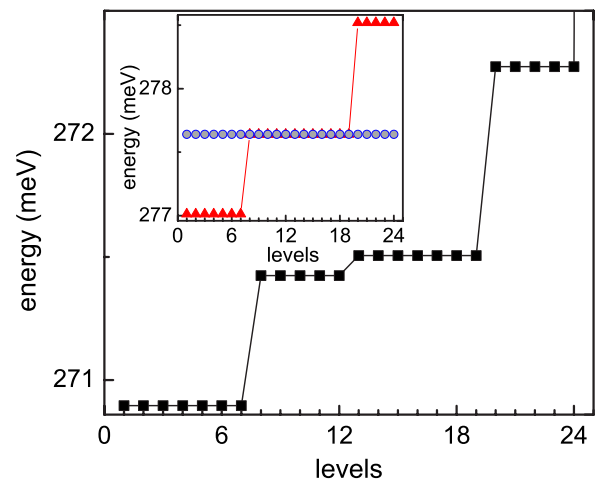

FIG. 5. (Color online) Energy spectrum of a three-electron quantum dot calculated for the case the magnetic impurity, is located at the position $\left(l_{0}, 0\right)$ and the Coulomb interaction strength is 0.5 . The inset is the low energy spectrum with (the red triangles) and without (the blue dots) the magnetic ion when the partially filled $p$ shell is considered.

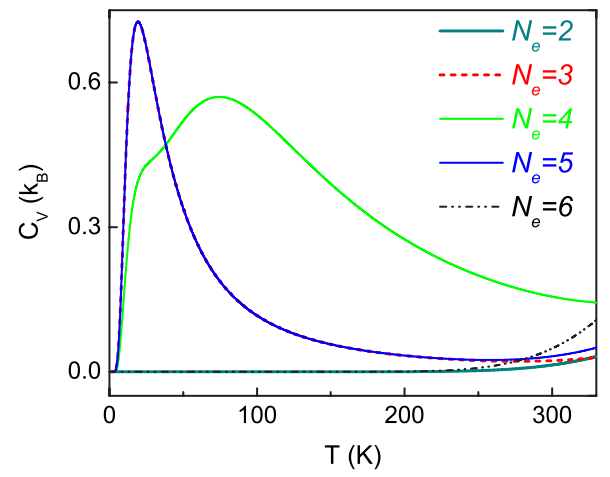

FIG. 6. (Color online) Heat capacities vs temperature plot for two to six electrons obtained for all possible configurations of $s, p$, and $d$ shells. The impurity was located at $\left(l_{0}, 0\right)$ and the Coulomb strength was 0.2 .

orbitals up to the partially filled $p$ shell are included. In this inset, we show also the spectrum as obtained without interaction with the magnetic impurity as the constant energy level (blue circles) with degeneracy 24 (four degenerate possibilities of the $p$ electron and six possibilities of the spin of the magnetic ion). In the presence of the magnetic impurity, the spectrum splits into three levels. The ground-state energy is shifted to lower energy with degeneracy 7 (spin degeneracy of the magnetic ion $5 / 2$ plus $1 / 2$ for the third electron) as indicated by the triangles, and the first excited state with degeneracy 12 equals to the ground-state energy of the case without interaction with the magnetic ion. This is a result of the intralevel interactions. When we include even more single-particle levels we obtain the results as given in the main plot of Fig. 5 where we took into account interlevel interactions, in this case $N_{s}=21$. Note that the ground-state energy is even further reduced in energy and the degeneracy of the excited state is further reduced. However, the groundstate energy in both cases has a degeneracy of 7 , which is related to the fact that the $\mathrm{Mn}$ spin is ferromagnetically coupled to the electron that couples to the $p$ shell. We see that the excited states split into more levels as compared to the case in the inset. The precious first excited states is split into a level with degeneracy 5 (=spin degeneracy due to total spin of $5 / 2-1 / 2=2$ ) and a level with degeneracy 7 . In the main figure we see several different energy gaps between the excited states. These gaps will have an effect on the contribution to the possible energy storage of the system that will be manifested in the heat capacity.

\section{HEAT CAPACITY}

Since we have the complete spectrum we can calculate all thermodynamic properties. One important quantity that is concerned with storage of energy is the heat capacity

$$
C_{V}\left(\lambda, T, R_{\mathrm{Mn}}\right)=\frac{\partial\left\langle E\left(\lambda, T, R_{\mathrm{Mn}}\right)\right\rangle}{\partial T},
$$

where 


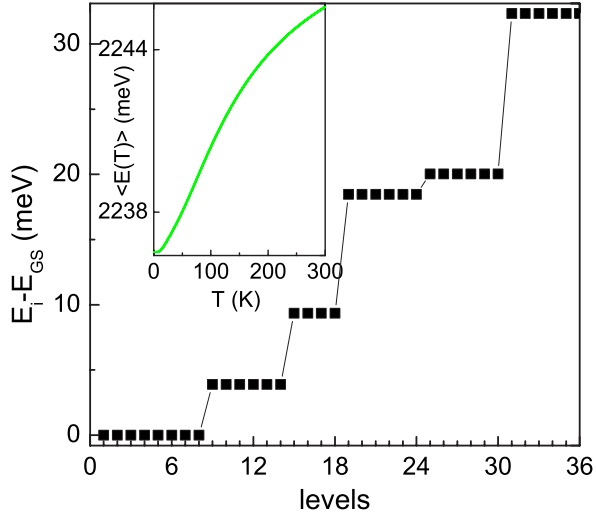

FIG. 7. (Color online) Spectrum of a four-electron quantum dot with the magnetic ion located at the position $\left(l_{0}, 0\right)$ when the three shells of $s, p$, and $d$ are included. The inset is the statistical average of the energy over temperature.

$$
\left\langle E\left(\lambda, T, R_{\mathrm{Mn}}\right)\right\rangle=\frac{\sum_{\alpha=1}^{N_{\alpha}} E_{\alpha} e^{-E_{\alpha} / k_{B} T}}{\sum_{\alpha=1}^{N_{\alpha}} e^{-E_{\alpha} / k_{B} T}}
$$

is the average energy that is a function of the Coulomb strength $\lambda$, temperature $T$, and the position of the impurity $R_{\mathrm{Mn}} . E_{\alpha}$ is the $\alpha$ th energy level of the many-electron energylevel spectrum.

We present in Fig. 6 the heat capacities of quantum dots containing two to six electrons. The impurity was located at the position $\left(l_{0}, 0\right)$. The heights and widths of the pronounced peaks of those curves are determined by the energy gaps between the respective ground-state and excited-state energies.

We plot in Fig. 7 the energy spectrum of the first 36 levels of the four-electron quantum dot in order to understand the heat capacity. The number of energy levels is relevant for the considered temperature range. From this figure we realize that the first peak in the heat capacity (see Fig. 6) is related to the turn of the averaged energy quantity (see the inset) at $T$ $=74.4 \mathrm{~K}$. The flatlike region in the average energy results in a zero heat capacity for temperatures close to zero. Besides,

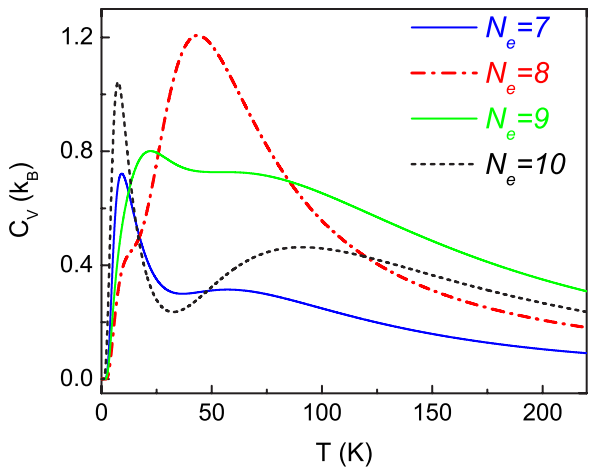

FIG. 8. (Color online) The same as Fig. 6 but obtained for seven to ten electrons.

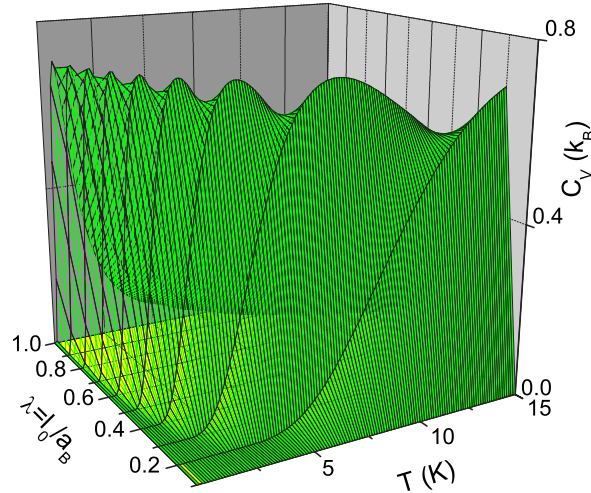

FIG. 9. (Color online) Three-dimensional (3D) plot of the heat capacity of the three-electron quantum dot containing a single impurity located at $\left(l_{0}, 0\right)$ vs temperature and Coulomb strength $\lambda$.

we see that the ground-state energy has degeneracy 8 resulting from the total spin of $5 / 2+1=7 / 2$ while the first excited state has degeneracy 6 that is from the total spin of $5 / 2+0$ $=5 / 2$, and so on for the higher energy levels.

In Fig. 8 we extend the result of Fig. 6 to $N_{e}=7-10$. Notice that for $N_{e}>4$ the heat capacity exhibits a clear peak structure for temperatures $T<100 \mathrm{~K}$. The position of the peak depends sensitively on the number of electrons in the dot.

Figure 9 shows a 3D plot of the heat capacity obtained as a function of temperature, $T$, and Coulomb strength, $\lambda$, of a three-electron quantum dot at a specific position of the magnetic impurity, $\left(l_{0}, 0\right)$, and shows clearly the effect of the Coulomb interactions. The result was obtained when all the possible electronic configurations of the $s, p, d, f$, and $g$ shells were included. We see a shift in the peak of the heat capacity to smaller temperatures when increasing the Coulomb interaction strength $\lambda$. Figure 10, plotted for two extremes (i.e., 0.1 and 1.0) of the Coulomb interaction strength used in Fig. 9, and it illustrates the respective energy spectra of these cases and their statistical average energies over temperature (the two insets). We see that at these two parameters

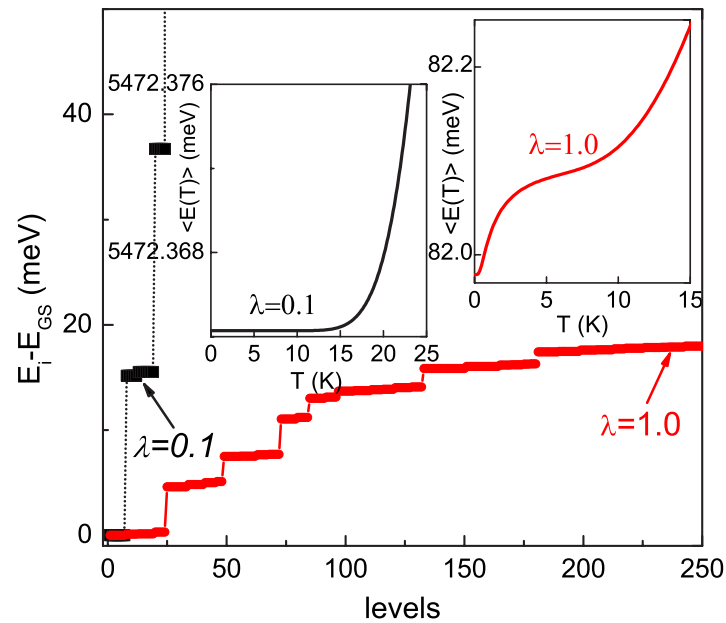

FIG. 10. (Color online) Spectrum of a three-electron quantum dot with the magnetic ion located at $\left(l_{0}, 0\right)$ for two values of the Coulomb interaction strength. 


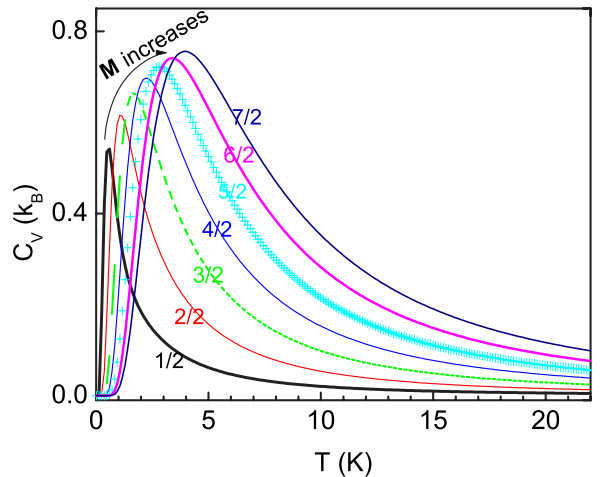

FIG. 11. (Color online) Heat capacity for $N_{e}=3$ and different values of the spin of the magnetic ion from $1 / 2$ to $7 / 2$. The impurity was located at $\left(l_{0}, 0\right)$ and the strength of the Coulomb interaction was 0.5 .

of $\lambda=0.1$ and $\lambda=1.0$, the respective energy spectra are significantly different explaining the shift in the peak structure of the specific heat. Because the peak of the specific heat is relevant to the gap between the energy levels and consequently to the temperature. If one plots the specific heat versus different Coulomb interaction strengths $\lambda$, which is proportional to $1 / \sqrt{\omega_{0}}$, and different temperatures one could see that the peak in the specific heat moves to lower temperature with increasing $\lambda$.

To complete the discussion on the heat capacity, let us discuss the dependence of the heat capacity on the spin size $\mathbf{M}$ of the magnetic impurity. Figure 11 shows that when the spin size of the impurity is changed from $1 / 2$ to $7 / 2$ the position of the peak in the heat capacity, along with its height and width, is shifted to higher temperatures. Notice that with increasing $\mathbf{M}$ the peak structure shifts to higher temperatures due to the increased interaction energy that leads to a larger gap in the energy spectrum.

\section{CONCLUSIONS}

In conclusion, we examined the behavior of few-electron quantum dots confined to a lateral parabolic potential in interaction with a single impurity in the absence of an external magnetic field. The impurity is engineered at different posi- tions. We produced results obtained for a few discrete positions and focused on three locations where the diagonal exchange matrix elements have a maximum: the center of the dot, the position $\left(l_{0}, 0\right)$, and the position $\left(\sqrt{2} l_{0}, 0\right)$. Results are intended to exhibit the electronic ground-state properties when the number of carriers is changed and many singleelectron levels are included due to a desire to sample as many electronic configurations as possible. We obtained different behaviors for different positions of the magnetic ion.

In fact, the exchange matrix elements (the electron-Mn interaction terms) are much smaller than $\hbar \omega_{0}$ (at most $1 \%-$ $2 \%$ ), particularly when we move the magnetic ion away from the center of the dot. The diagonal elements become dominant in comparison to contributions from the exchange interaction terms. Therefore, $\Delta_{Z}$ is of the same order as those small parameters.

Extending the Hilbert space allows us to understand physics beyond the $s, p$, and $d$ shell interactions and we can study the properties in the case of larger Coulomb interaction strength. We found that $\Delta_{Z}$ is related to the Coulomb strength $\lambda$ and the position of the magnetic ion. $\lambda$ can, for example, be changed by changing the size of the quantum dot.

When $\lambda$ is small, meaning that the electrons in the quantum dot are more strongly localized, $\Delta_{Z}$ changes consistently with changes in the diagonal electron-Mn exchange matrix elements. In contrast, when $\lambda$ is large the electrons in the quantum dot become more strongly correlated. The contribution to the energy of the system spreads over more matrix elements, and $\Delta_{Z}$ becomes maximum at different positions of the Mn impurity.

With changing number of electrons in the quantum dot system and changing position of the magnetic ion result in changes in the heat capacity both in its shape and the position of the peaks. Larger Coulomb interaction strength moves the peak of the heat capacity to lower temperature.

\section{ACKNOWLEDGMENTS}

We acknowledge fruitful discussions with M. B. Tavernier. This work was supported by FWO-Vl (Flemish Science Foundation), the EU network of excellence SANDIE, and IAP (Belgian Science Policy).

\footnotetext{
*nga.nguyen@ua.ac.be

†rancois.peeters@ua.ac.be

${ }^{1}$ L. Jacak, P. Hawrylak, and A. Wojs, Quantum Dots (Springer, Berlin, 1997).

${ }^{2}$ P. A. Maksym, Phys. Rev. B 53, 10871 (1996).

${ }^{3}$ U. Merkt, J. Huser, and M. Wagner, Phys. Rev. B 43, 7320 (1991).

${ }^{4}$ G. Bacher et al., Phys. Status Solidi B 224, 573 (2001).

${ }^{5}$ L. Besombes, Y. Léger, L. Maingault, D. Ferrand, H. Mariette, and J. Cibert, Phys. Rev. Lett. 93, 207403 (2004); Y. Léger, L. Besombes, L. Maingault, D. Ferrand, and H. Mariette, ibid. 95, 047403 (2005); Phys. Rev. B 72, 241309(R) (2005).
}

${ }^{6}$ L. Maingault, L. Besombes, Y. Léger, C. Bougerol, and H. Mariette, Appl. Phys. Lett. 89, 193109 (2006); Y. Léger, L. Besombes, J. Fernández-Rossier, L. Maingault, and H. Mariette, Phys. Rev. Lett. 97, 107401 (2006).

${ }^{7}$ K. Chang, J. B. Xia, and F. M. Peeters, Appl. Phys. Lett. 82, 2661 (2003).

${ }^{8}$ J. Fernández-Rossier and L. Brey, Phys. Rev. Lett. 93, 117201 (2004).

${ }^{9}$ A. O. Govorov, Phys. Rev. B 72, 075358 (2005).

${ }^{10}$ Fanyao Qu and Pawel Hawrylak, Phys. Rev. Lett. 95, 217206 (2005).

${ }^{11}$ J. K. Furdyna, J. Appl. Phys. 64, R29 (1988). 
${ }^{12}$ A. L. Efros, M. Rosen, and E. I. Rashba, Phys. Rev. Lett. 87, 206601 (2001); A. K. Bhattacharjee and J. Pérez-Conde, Phys. Rev. B 68, 045303 (2003); J. Fernández-Rossier, ibid. 73, 045301 (2006); J. Fernández-Rossier and Ramón Aguado, Phys. Rev. Lett. 98, 106805 (2007).

${ }^{13}$ F. M. Peeters and V. A. Schweigert, Phys. Rev. B 53, 1468
(1996).

${ }^{14}$ N. F. Mott, Metal-Insulator Transitions (Taylor and Francis, London, 1990).

${ }^{15}$ E. V. Tsiper, J. Math. Phys. 43, 1664 (2002).

${ }^{16}$ M. B. Tavernier, E. Anisimovas, F. M. Peeters, B. Szafran, J. Adamowski, and S. Bednarek, Phys. Rev. B 68, 205305 (2003). 